\title{
Birth of Axiomatization Definition of Probability Theory Liqiang Chen
}

\author{
Ankang University, Ankang, 725000, China
}

\begin{abstract}
Key words: Classical probability, Analytical probability, Axiomatized probability
\end{abstract}
\begin{abstract}
The discipline of probability theory is an ancient and young discipline. Its development experienced three periods: classical probability period, analytical probability period and axiomatized probability period. This paper analyzed development features of probability theory in these three stages and summarizes the appearance and results of axiomatized probability.
\end{abstract}

\section{Introduction}

Probability theory is a typical mathematics discipline, which is beyond all doubt. But before 1930s, nobody dared to say this, even if great mathematician did not dare to say this. This is because discipline development was not perfect. Through development for several hundred of years (about 300 years since its birth) and efforts of several generations of people, probability theory also gained certain progress, but its foundation was not so rigorous like other certain mathematics. What is the problem? New era development calls for new mathematics researches. Under such background, a great Soviet mathematician A.N.Kolmogorov (English name) /Андрей Николаевич Колмогоров (Russian name) (April 25, 1903 - October 20, 1987)summarized predecessors' work and introduced set theory, measure theory and other modern mathematics views in probability theory. Since then, probability theory has gained brand-ne wand long-term development and gradually become a rigorous mathematics branch (random mathematics) like other mathematics discipline.

\section{Introduction to development course of probability theory}

\section{Birth of probability theory}

In the probability theory, it is believed that probability theory was born in 1654 .

In 1494, Italian mathematician Luca Pacioli (about 1445 - 1517) proposed in his works Abstract of Arithmetic, Geometry, Ratio and Proportion that two gamblers gamble several rounds and the one who first wins $x$ rounds will win. Now, A wins $m$ rounds $(m<x)$ and $B$ wins $n$ rounds $(n<x)$. However, due to an irresistible cause, the gambling party is force dot terminate. The problem is that, how should the bet be distributed fairly? This is famous "bet distribution problem" (also called "the problem of points”). This problem was studied repeatedly in detail and discussed for over 200 years. Finally, French mathematician B. Pascal (1623-1662) and P.de Fermat (1601-1665) solved this problem. In 1654, the gambler $A \cdot G \cdot G \cdot d e$ Mere (1610-1685) consulted "the problem of points" to B.Pascal. B.Pascal and P.de Fermat wrote 7 letters to each other. The third letter was regarded by mathematics historian as the date when probability theory was born [1]. The core value of the two masters is that they studied probability theory (random mathematics) by mathematic means the first time. The real probability theory works is On Calculation in Gambling which was written by Christian Huygens (1629-1695) (Dutch mathematician, physicist and astronomer) and published in 1657. It is 22 years earlier than the time - 1679 when the letters between B.Pascal. B.Pascal and P.de Fermat were published. This book is the first monograph about probability theory which systematizes, formulizes and generalizes the knowledge of probability theory. It was once widely used as a standard teaching material of probability theory in Europe. The above three jointly serve as the founders of probability theory. 


\section{Classical probability period}

In Europe in the $17^{\text {th }}$ century, gamblers often played the game of shooting dice. According to his long-term gambling experience, $A \cdot G \cdot G \cdot$ de Mere (as mentioned above) considered the possibility that a 6-point appeared when a dice was shot for 4 times was larger than the possibility that a pair of 6-point appeared when two dices were shot for 24 times. Actually, the probability of the former is $1-\left(\frac{5}{6}\right)^{4}=0.5177$, and the probability of the latter is $1-\left(\frac{35}{36}\right)^{4}=0.4614$, . There is tiny difference. Mere's guess is the right, but he did not calculate, but from his long-term gambling experience [2]. This also reveals his exquisite gambling technique. We can also know gambling prevailed in Europe. All these offer valuable materials for development of probability theory. Classical probability theory is as follows: a. total result of random experiment is known in advance - the number of sample points in sample space is given; b. the sample space of each result is equal. For example, the capacity of sample space ( $\Omega$ ) is $\mathrm{m}$, event $\mathrm{A} \subset \Omega$; event A contains n sample points; the occurrence probability of event $A$ in a random experiment is $P_{m}^{n}(A)=\frac{n}{m} \in[0,1]$. The former: the probability that 6-point does not appear in each experiment is $\frac{5}{6}$; the probability that 6-point does not appear in 4 times is $\left(\frac{5}{6}\right)^{4}$ once in 4 experiments; similarly, the latter can be calculated.

In fact, geometric probability is the extension of a guess of classical probability. Under the mathematics background, this could not be proven. French mathematicians $G \cdot L \cdot L \cdot$ Buffon (1707-1788), A·De Moivre (1667-1754), P·S·M·de Laplace (1749-1827), S·D· Poisson (1781-1840) and German mathematician Carl Friedrich Gauss first carried out the work in this aspect. For example, Probability Experiment published by Buffon in 1777 first proposed and solved "Buffon needle test" problem ("Buffon needle test" is a modern term; actually, it is geometric probability). The publishing of Art of Conjecturing by Swiss mathematician Jacob Bernoulli (1654-1705) means the ending of lengthy probability theory formation process and the beginning of mathematical probability theory. A De Moivre mentioned multiplication formula of independent events the first time in Opportunity Theory published in 1718, gave binomial distribution formula and discussed "the problem of points". Gauss first found normal distribution from the perspective of error function in 1809. This finding made mathematical statistics in the $19^{\text {th }}$ century become the era ruled by normal distribution. But, many classical problems were not solved. The thought of probability theory was not systematized and perfected. The era development promoted the emergence of new theories.

\section{Analytical probability period}

I·Newton (1643-1727) and Gottfried Wilhelm Leibniz (1646-1716) created calculus. Mathematicians in the $18^{\text {th }}$ century utilized calculus to study each field and gained great achievements. Hence, mathematical analysis obtained great development. Differential equation, characteristic function, inversion formula and moment generating function gradually became mathematical tools to study probability theory. A symbolic works in his period is Theory of Probability Analysis of Laplace in 1812.

This works coverall summarized research achievements of probability theory in the $18^{\text {th }}$ century. The contents include: geometric probability, Bernoulli Theorem and least square method etc. besides, the works specified the definition of classical probability. $\mathrm{n}$ ! asymptotic formula $n ! \approx \sqrt{2 \pi} n^{n+\frac{1}{2}} e^{-n}$ proved A.De Moivre- Laplace central-limit theorem when the probability is 0.5 . He is the first person laying the foundation of probability theory in a rigorous and systematic way. An important shortcoming is that he wanted to bring any probability problem into simple classical probability model. This is vain, obviously. 
In the second half of $19^{\text {th }}$ century, probability theory gained new development. Some Russian mathematicians called Moscow School studied it. Nikolas lvanovich Lobachevsky (1792-1856) is the first person. He mainly studied noneuclidean geometry and did not study probability theory. He generated interest in probability theory, because it would be used in geometry. He had twp papers about probability theory.

Виктор Яковлевич Буняковский (1804-1889) published the first probability theory works in 1846 - Foundation of Mathematics Probability Theory in Russia. This book quoted the wrong view of "science of ethics" due to the influence of Laplace and Poisson, but this book still has great significance. It introduced the results of probability theory in Europe the first time. Till now, probability theory terms quoted by Виктор Яковлевич Буняковский are still used.

One of Виктор Яковлевич Буняковский’s students isПафну́тий Льво́вич Чебышёв (1821.5.16-1894.12.8) (also translated to Chebyshev in some books). He just published 4 papers about probability theory, but they generated long-term effects on the development of probability theory, such as Chebyshev Inequality and Chebyshev Law of Large Numbers. His thought of probability theory laid theoretical basis for the formation of Moscow School. The representatives of this school include МарКОВ, АНдрей АНдрееВич (1856.6.14-1922.7.20), Ляпунов (1857.6.6-1918.11.3), Aleksandr Yakovlevich Khinchin (1894-1959) and Андрей Николаевич Колмогоров (1903.4.25-1987.10.20). The first two mainly studied independent random variables sequence and Markov chain probability model. The latter two mainly introduced the view of functions of real variable in probability theory. МарКОВ, АНдрей АНдрееВич and Ляпунов are the students of Chebyshev. МарКОВ, АНдрей АНдрееВич set up Markov chain theory. This theory later independently developed into a discipline - random process. Ляпунов utilized his own invention characteristic function to prove Lyapunov central-limit theorem. Characteristic function later became one of important tools to study probability theory.

\section{Birth of "axiomatization" definition of probability theory}

\section{Rigorous demand for the definition of probability theory}

Since the $20^{\text {th }}$ century, mathematics disciplines except probability theory have gained rapid development. The definition of probability theory was still equivocal. Unclear definition strictly restricted its development. "Bertrand's paradox" is an indispensible example in the development of probability theory. J Bertrand (1822-1900) is a French mathematician. He proposed to consider an equilateral triangle inscribed with a circle in Probability Theory. If a string on the circle is randomly chosen, what is the probability that the length of this string is longer than the side length of the triangle [3]? Bertrand gave three solutions and obtained three different results. This event indicates if probability theory wanted to continue to develop in the $20^{\text {th }}$ century, its concept must be perfected.

\section{Several trials of rigorous requirements for probability theory definition}

At the beginning of $20^{\text {th }}$ century, many mathematicians tried to solve this problem. Russian mathematician Bernstein (Серге́й Ната́нович Бернште́йн, 1880.3.5-1968.10.26) first tried the concept of probability theory. 1917, Bernstein published a paper with the title of "On Axiomatization Foundation of Probability Theory". In subsequent years, he was still devoted to studying axiomatization of probability theory. In 1927, his book Probability Theory came out. Bernstein offered a detailed probability theory axiomatization system. His three major axioms include: a. probability comparability axiom; b. incompatible event axiom; c. event combination axiom. Based on the three axioms, Bernstein first systematically constructed the whole probability theory building. However, he established it based on qualitative comparison of random event probability and events. Even probability is numerical value deduced, rather than real concept. So, Bernstein's work fails to really solve axiomatization definition of probability theory.

In this period, another mathematician making significant contributions to axiomatization of probability theory was Austrian R·von Mises (1883.4.19-1953.7.14). His main work was frequency definition of probability theory and axiomatization of statistics definition. In his book Probability, 
Statistics and Truth written in 1928, he established limit theory of frequency and emphasized that probability concept was significant only when a large number of phenomena existed. Although frequency definition of probability is easy to understand visually, the probability that an event appears countless times under independent repeated experiments will not be handled by Mises theory. Without new theory, the definition of probability theory is still vague. Thus, many calculations of probability theory cannot be correctly completed. The era calls for new probability theory system.

\section{Final establishment of probability theory axiomatization definition}

French mathematician E Borel (1781-1956) first introduced measure theory in the research of probability theory. His work indicated a new research road for mathematicians to study probability theory. The achievements of soviet mathematician A.N. Kolmogorov (as mentioned above) are quite outstanding. After he deduced necessary and sufficient conditions of weak law of large numbers in 1926, he gave the most general result for strong large number theorem proposed by Borel. Thus, one of the central subjects - law of large numbers in probability theory was completed. This became the prelude of probability theory axiomatization based on measure theory [4].

In 1933, A.N. Kolmogorov published Foundations of Probability Theory of German version. This is a milestone event. In the book, he established probability theory axiomatization system based on measure theory and laid the foundation of modern probability theory. Besides, analogy of set measure and event probability, analogy of function orthogonality and random variable independence and analogy of mathematical expectation and integral were set up in the book. These analogies give probability theory the feature of deductive mathematics [5].

There are 5 initial conditions of A.N. Kolmogorov's axiomatization. The whole probability theory building develops on the basis of the 5 axioms. Kolmogorov's work gained the approval of more and more mathematicians so that probability theory transformed into mathematics foundation with rigorous foundation like other mathematics branches from scientific foundation with semi-physical property. Set theory and measure theory play an indispensible role in the process of probability theory axiomatization.

\section{Achievements after the establishment of axiomatization definition of probability theory}

Based on axiomatization definition of probability theory, modern probability theory gains a series of achievements. In 1931, Kolmogorov laid theoretical foundation of a kind of common random processes - Markoff process with analysis method. After Kolmogorov, the scientists making great contributions to axiomatization system of probability theory include: French mathematician Paul Pierre Levy (1886-1971), Soviet mathematician Khinchin 9as mentioned above), American mathematician Joseph Leo Doob (1910.10.27-2004.6.7) and Japanese mathematician Kyoshi Ito (1915.9.7-2008.11.10). In 1948, Paul Pierre Levy published the works Random Process and Brownian Movement, put forward general theory of independent increment process and greatly drove development of special Markov chain - Brownian movement. In 1933, the theories related to stationary process were proposed. In 1939, Paul Pierre Levy introduced the concept of "martingale”. In 1950, Joseph Leo Doob studied martingale system so that martingale became an independent branch - martingale theory. From 1942, Japanese mathematician Kyoshi Ito established stochastic integral and stochastic integral. His theory laid a foundation for creating and developing this new branch in stochastic analysis while opening up a new research direction of random process.

\section{Conclusion}

In the whole development history of probability theory, axiomatization system of probability theory is a mathematical thing that those studying random mathematics contact all day long. It's necessary for us to make it clear. The world is developing, and probability theory system is also developing. At present, axiomatized probability theory system is also the most advanced theory. The development of related discipline it brings is obvious to all. We cannot predict the future, but in a short term, axiomatized probability theory is a theoretical foundation to study random mathematics. 


\section{Acknowledgments}

This paper is a part of high-level talents project in Ankang University, No. 2013AYQDZR11

\section{References}

[1] Yang Jing, Xu Chuansheng, Development of mathematics technology and probability theory. Journal of Taiyuan University of Technology (Social Sciences), 2008-3: Vol.26 No.1.

[2] Lai Jingyao, Origin and development of probability theory. Journal of Northwest Normal University, 1984-3: 10

[3] Cheng Xiaohong, Yang Jing, Exploration of source of probability theory axiomatization. Journal of Northwest University (Natural Science Edition), 2007-12, Vol 37,No.6

[4] Zhu Chunhao, Concise outline of academic history of probability theory. Journal of Wuhan Institute of Shipbuilding Technology, 2010-5: 105

[5] Li Wenlin, Course of history of mathematics. Beijing: Higher Education Press, 2000.290. 